\title{
Three-Dimensional Crack Analysis using X-FEM Considering Symmetric Conditions"
}

\author{
Toshio NAGASHIMA ${ }^{* *}$ and Naoki MIURA *** \\ ${ }^{* *}$ Faculty of Science and Technology, Sophia University \\ 7-1 Kioicho, Chiyoda-ku, Tokyo, 102-8554, JAPAN \\ E-mail: nagashim@me.sophia.ac.jp \\ ${ }^{* * *}$ Central Research Institute of Electric Power Industry \\ 2-11-1 Iwado Kita, Komae-shi, Tokyo 201-8511, JAPAN \\ E-mail: miura@criepi.denken.or.jp
}

\begin{abstract}
The extended finite element method (X-FEM), which can model the domain without explicitly meshing the crack surface, can be used to perform stress analyses for efficiently solving fracture mechanics problems. In the present study, the constraint condition enforcement for X-FEM analysis considering symmetry is presented. Since the interpolation functions utilized in X-FEM analysis include the enrichment basis functions, the freedoms of the node on the symmetric plane should be constrained properly in the X-FEM model with symmetric conditions. Moreover, evaluation of the energy release rate by the domain integral method should be performed considering the symmetry conditions. In the present paper, the constraint conditions for three-dimensional X-FEM analysis considering symmetric conditions are summarized, and numerical examples using symmetric X-FEM models are shown. The proposed procedure can be used to perform efficient $\mathrm{X}$-FEM analyses of practical fracture problems.
\end{abstract}

Key words: Extended FEM, Fracture Mechanics, Energy Release Rate, Symmetric Conditions

\section{Introduction}

The extended finite element method (X-FEM) ${ }^{(1)-(3)}$ can approximate the discontinuous displacement field near cracks independently of the finite element mesh by using the interpolation functions, which can describe the displacement field near cracks in structures. Therefore, the crack modeling for stress analyses can be performed more easily by X-FEM, as compared to conventional FEM. As the information with respect to the crack geometry is required in order to determine the interpolation functions in X-FEM, the level set method ${ }^{(4)}$, which expresses the geometry implicitly as the zero contour of the level set function, can be used in order to simplify the computation process in X-FEM analysis ${ }^{(5)}$. The X-FEM analyses in conjunction with the level set method ${ }^{(6)}$ have been applied to the three-dimensional elastic stress and crack propagation analyses for planar and non-planar crack with complex geometry ${ }^{(7)}{ }^{(8)}$. Moreover, X-FEM has come to be applied to geometrically nonlinear ${ }^{(9)(10)}$ and materially nonlinear problems ${ }^{(10)-(13)}$. In elastic problems, the energy release rate, which is one of fracture mechanics parameters, can be evaluated by the post processing for the results of X-FEM analysis, as well as FEM. Since the crack geometry does not always perfectly coincide with the element boundary in X-FEM models, neither the virtual crack closure method ${ }^{(14)}$ nor the virtual crack extension method ${ }^{(15)(16)}$ can be applied directly. Therefore, the domain integral method ${ }^{(17)(18)}$ is used to evaluate the

Received 22 Feb 2008 (No. T1-05-1069) Japanese Original : Trans. Jpn. Soc. Mech. Eng., Vol.72, No.719, A (2006), pp.974-981 (Received 6 Oct., 2005) [DOI: 10.1299/jcst.2.210] 
energy release rate ${ }^{(1)-(3),(6)-(8)}$.

The symmetrical conditions can be considered in order to reduce the number of nodes and elements and perform effective analyses in X-FEM as well as FEM when the geometry and boundary conditions have the symmetry properties. However, because the interpolation functions utilized in X-FEM analyses include the enrichment basis functions and the freedoms with respect to the enrichment basis are added to the normal nodal freedoms, the constrained conditions are given carefully for nodes on the symmetrical plane. The establishment of a rational method to enforce symmetric conditions appears to be indispensable for practical X-FEM analyses. However, the literature contains no numerical results obtained by X-FEM considering the symmetric conditions. Therefore, the present study considers a rational method by which to enforce symmetric boundary conditions in $\mathrm{X}$-FEM analysis. In addition, a method by which to use the symmetric conditions in order to evaluate the energy release rate by using the domain integral method as post processing of $\mathrm{X}-\mathrm{FEM}$ analyses is discussed. Moreover, the proposed method to enforce the symmetric condition is verified through the numerical analyses of three-dimensional crack problems using X-FEM.

\section{Introduction}

In Section 2, X-FEM, which is used to perform small deformation linear elastic analyses for three-dimensional structures including planar cracks, is outlined, and the domain integral method, which is used to evaluate the energy release rate at the crack front by post processing of the stress analysis results, is described. Eight-node hexahedral linear finite elements are used in the three-dimensional X-FEM analysis.

\subsection{Interpolation function}

As shown in Fig. 1, the analyzed domain is defined in Cartesian coordinates $(x, y, z)$ and the planar crack is assumed to be on the $x-y$ plane. In X-FEM, the approximate displacement function $\boldsymbol{u}^{\mathrm{h}}$ of the distributed displacement $\boldsymbol{u}$ near a planar crack is expressed as:

$$
\begin{aligned}
& \mathbf{u}^{h}(\mathbf{x})=\sum_{I=1}^{8} N_{I}(\mathbf{x}) \mathbf{u}_{I}+\sum_{I \in \mathbf{C}} N_{I}(\mathbf{x}) \sum_{k=1}^{4} \gamma_{k}(\bar{g}(\mathbf{x}), z) \mathbf{a}_{I}^{k}+\sum_{I \in \mathbf{J}} N_{I}(\mathbf{x}) H(z) \mathbf{b}_{I} \\
& \bar{g}(\mathbf{x})=\sum_{I=1}^{8} N_{I}(\mathbf{x}) g\left(\mathbf{x}_{I}\right)
\end{aligned}
$$

where $N_{\mathrm{I}}$ is the interpolation function used in the formulation of the conventional FEM, $\boldsymbol{C}$ and $\boldsymbol{J}$ denote the node set considering the asymptotic solution $\gamma_{\mathrm{i}}(\mathrm{i}=1 \ldots 4)$ and the Heaviside function $H(x)$ near a crack, respectively, and $\boldsymbol{u}_{\boldsymbol{I}}, \boldsymbol{a}^{\boldsymbol{k}}{ }_{I}$ and $\boldsymbol{b}_{\boldsymbol{I}}$ denote the vectors of freedom assigned to each node. Here, $\boldsymbol{C} \cap \boldsymbol{J}=\boldsymbol{\phi}$ is satisfied. $g(\mathbf{x})$ is a level set function introduced in order to express the shape of the front tip of the crack using nodal information. The value of the level set function $\bar{g}(\mathbf{x})$ is interpolated using the nodal value of the level set function.

The level set function $g(\mathbf{x})$ is described as follows:

$$
g(\mathbf{x})=\min _{\overline{\mathbf{x}} \in \Gamma_{t}}\|\mathbf{x}-\overline{\mathbf{x}}\| \operatorname{sign}\left(\mathbf{n}(\overline{\mathbf{x}})^{T}(\mathbf{x}-\overline{\mathbf{x}})\right)
$$

where $\Gamma_{t}$ represents the curved front line of the crack, and $\mathbf{n}(\overline{\mathbf{x}})$ denotes the unit vector at point $\overline{\mathbf{x}}$ orthogonal to the curved line $\Gamma_{t}$ on the $x-y$ plane.

As shown in Fig. 1, the absolute value of $g$ is the distance between the projected point on the crack surface ( $x-y$ plane) and the crack front. The sign of $g$ indicates the location with respect to the curved crack front, where the level set function is negative if the projected point is located inside the crack and is positive if the projected point is located in a ligament. 
In the present study, the near-tip functions, which can reconstruct the asymptotic displacement field near a crack tip in a homogeneous isotropic material in a two-dimensional elastic body, are used and are defined as follows:

$$
\gamma_{1}=\sqrt{r} \cos \left(\frac{\theta}{2}\right), \gamma_{2}=\sqrt{r} \sin \left(\frac{\theta}{2}\right), \gamma_{3}=\sqrt{r} \sin \left(\frac{\theta}{2}\right) \sin \theta, \gamma_{4}=\sqrt{r} \cos \left(\frac{\theta}{2}\right) \sin \theta
$$

where $r$ and $\theta$ are the polar coordinates in a plane defined near the crack tip, and

$$
\begin{aligned}
& r=\sqrt{g^{2}+z^{2}} \\
& \theta=\arctan (z / g)
\end{aligned}
$$

In three-dimensional problems, for Eq. (1a), the degrees of freedom $\mathbf{a}^{k}{ }_{J}$ with respect to the enrichment nodes with asymptotic basis functions, the number of which is $3 \times 4=12$, and the three degrees of freedom $\boldsymbol{b}_{\boldsymbol{I}}$ with respect to the enrichment nodes with the Heaviside function, are added to the degrees of freedom used in the conventional FEM.

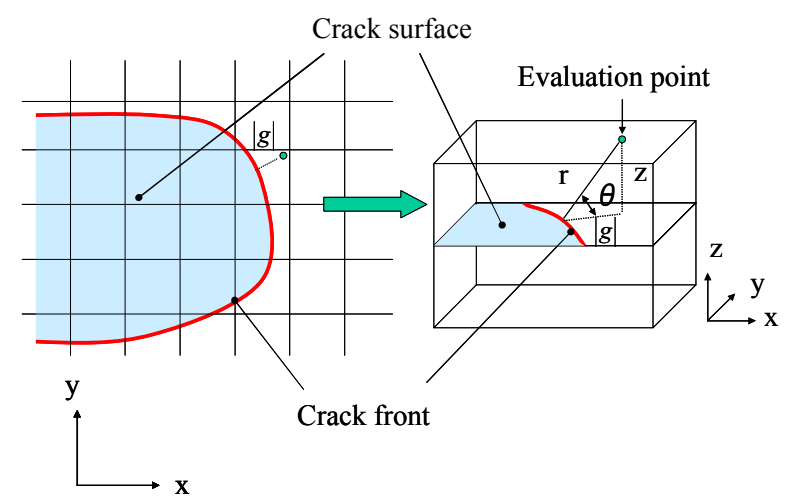

Fig. 1 X-FEM modeling for a planar crack

\subsection{Three-dimensional crack modeling using X-FEM}

The method used to model the opening crack by X-FEM is illustrated in Fig. 2. In the figure, the node labeled " $J$ " has an additional freedom for the Heaviside function, which can express the discontinuous displacement field near a crack surface, as shown in the third term of the RHS of Eq. (1a). The node labeled " $C$ " has an additional freedom for the asymptotic basis functions. If the crack front matches the element boundary perfectly, then Pattern-J can be used. Otherwise, the Heaviside function alone cannot express exactly the discontinuous displacement field near a crack front. In this case, Pattern-JC, in which the asymptotic basis functions are enriched, should be used.

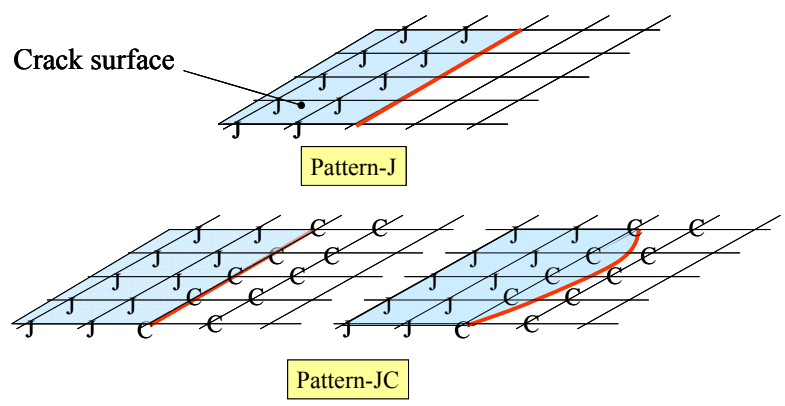

$C$ : Enriched node with asymptotic basic function

$J$ : Enriched node with the Heaviside function

Fig.2 Modeling of a planar crack by enrichment nodes 


\subsection{Numerical integration}

Since the interpolation function in the element, which is constructed from enriched nodes, includes high-order functions, the normal order of the Gauss numerical integration, such as the two-point Gauss integration formula utilized for an eight-node hexahedral linear element, may be inadequate with respect to accuracy. In X-FEM analysis, if an element includes a discontinuity displacement field, i.e., a bi-material interface or a free surface, then the element is partitioned into multiple domains and numerical integration is performed for each domain. However, in the present study, a higher order Gauss numerical integration is used to evaluate element stiffness for elements with enriched nodes.

\subsection{Evaluation of energy release rate}

In the finite element models used in X-FEM analysis, the geometries of the crack line for two-dimensional problems and the crack front for three-dimensional problems do not always match the element boundary. Therefore, the virtual crack extension method ${ }^{(14)}$ and the virtual crack closure method ${ }^{(15)(16)}$, which are used in conjunction with conventional FEM analysis, cannot be applied to evaluate the energy release rate. The domain form of the energy release rate ${ }^{(1)-(3)(6)-(8)}$ can be used to compute the energy release rate in conjunction with X-FEM. If we define the Cartesian local coordinate $(\tilde{x}, \widetilde{y}, z)$ such that the direction matches that of the crack extension, as shown in Fig. 3, the domain form of the energy release rate $G$ in three-dimensional problems can be expressed as follows:

$$
\begin{aligned}
& \bar{G}=\int_{V}\left[\left(\sigma_{\widetilde{x}} \frac{\partial u_{\tilde{x}}}{\partial \widetilde{x}}+\tau_{\widetilde{x} \tilde{y}} \frac{\partial u_{\tilde{y}}}{\partial \widetilde{x}}+\tau_{\widetilde{x} z} \frac{\partial u_{z}}{\partial \widetilde{x}}-w\right) \frac{\partial q}{\partial \widetilde{x}}+\left(\tau_{\widetilde{x} \tilde{y}} \frac{\partial u_{\widetilde{x}}}{\partial \widetilde{x}}+\sigma_{\tilde{y}} \frac{\partial u_{\tilde{y}}}{\partial \widetilde{x}}+\tau_{\widetilde{y} z} \frac{\partial u_{z}}{\partial \widetilde{x}}\right) \frac{\partial q}{\partial \widetilde{y}}\right. \\
& \left.+\left(\tau_{\widetilde{x} z} \frac{\partial u_{\widetilde{x}}}{\partial \widetilde{x}}+\tau_{\widetilde{y} z} \frac{\partial u_{\widetilde{y}}}{\partial \widetilde{x}}+\sigma_{z} \frac{\partial u_{z}}{\partial \widetilde{x}}\right) \frac{\partial q}{\partial z}\right] d V \\
& G=\frac{\bar{G}}{\int_{L_{c}} \alpha d C}
\end{aligned}
$$

where $\alpha$ is a scalar field that takes the value of one at the evaluation point and vanishes at the surface of the volume $V, L_{c}$ is the crack front, and $\sigma_{\mathrm{ij}}, u_{\mathrm{j}}$, and $w$ are the stress, displacement, and strain energy density, respectively.

In the present study, based on a study by Sukumar et al. ${ }^{(3)}$, a rectangular parallelepiped region defined in local Cartesian coordinates $(\tilde{x}, \tilde{y}, z)$, the center of which corresponds to the evaluation point at the front tip, and the evaluation points for stress, strain, and displacement are set in the region as shown in Fig. 4. The weight function $q$ takes the value of one at the evaluation point and vanishes at the surface. The weight function $q$ is expressed as follows:

$$
q(\tilde{x}, \tilde{y}, z)=\left(1-\frac{2 \tilde{x}}{L_{\tilde{x}}}\right)\left(1-\frac{2 \tilde{y}}{L_{\tilde{y}}}\right)\left(1-\frac{2 z}{L_{z}}\right)
$$

where $L_{\mathrm{x}}, L_{\mathrm{y}}$, and $L_{\mathrm{z}}$ are the lengths of the edges of a parallelepiped in the $\tilde{x}, \tilde{y}$, and $z$ directions, respectively.

The volume integration of Eq. (5a) can be performed numerically using several integration points inside the rectangular parallelepiped.

In the calculation of the energy release rate for three-dimensional crack problems, the direction of the crack extension is usually assumed to be perpendicular to the crack front in the crack surface. Therefore, if the geometry of the crack front is not linear, the direction of the crack extension generally varies depending on the geometry of the front tip. It is possible to specify the direction of the crack extension by describing it explicitly in the input data. However, in the present study, the direction is computed automatically using the level set value ${ }^{(7)}$. This method can easily determine the direction of the crack extension at 
any specified point on the crack front tip. More specifically, as shown in Fig. 5, the direction of the crack extension $\boldsymbol{n}$ should be orthogonal to that of the tangent to the curved crack front tip. The direction $\mathrm{n}$ can be determined by the equation, including the derivatives of the level set function, as follows:

$$
\mathbf{n}=\frac{1}{\sqrt{g_{x}{ }^{2}+g_{y}{ }^{2}}}\left(\begin{array}{l}
g_{x} \\
g_{y}
\end{array}\right)
$$

where

$$
g_{x} \equiv \frac{\partial g}{\partial x}, g_{y} \equiv \frac{\partial g}{\partial y}
$$

Since the level set function $g$ can be interpolated in each element using Eq. (1b), the derivative of the level set function is not continuous at the interface of the adjacent elements. Therefore, in practical calculations, $g_{\mathrm{x}}$ and $g_{\mathrm{y}}$ are averaged over each node so that averaged nodal derivatives of the level set function $g_{\mathrm{xI}}$ and $g_{\mathrm{yI}}$ can be obtained. The derivatives of the level set function can be interpolated using the following equations:

$$
\begin{aligned}
& g_{x}(\mathbf{x})=\sum_{I=1}^{8} N_{I}(\mathbf{x}) g_{x I} \\
& g_{y}(\mathbf{x})=\sum_{I=1}^{8} N_{I}(\mathbf{x}) g_{y I}
\end{aligned}
$$

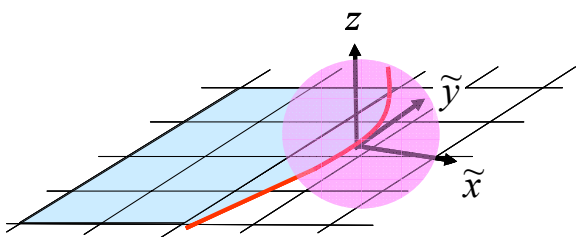

Fig. 3 Evaluation of energy release rate using the domain integral method for 3D crack

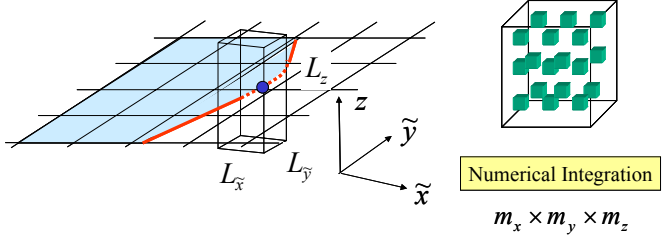

Integration point

Fig. 4 Volume for domain integral to evaluate energy release rate in 3D X-FEM analysis

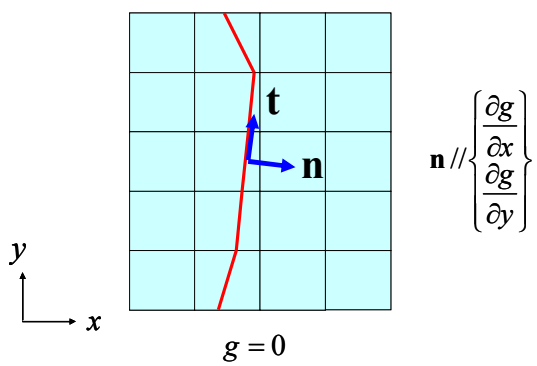

Fig.5 Modeling of a front geometry using level set method

\section{Introduction of symmetric conditions}

In Section 3, the method used to enforce the symmetric conditions for three-dimensional problems including planar cracks solved by X-FEM is presented. For simplicity, the method is first applied to a two-dimensional problem. Moreover, the domain integral method used to evaluate the energy release rate considering the symmetric condition is discussed. 


\subsection{Method to enforce the symmetric condition}

Here, using the example of a two-dimensional problem, the method to enforce the symmetric conditions for crack analyses by X-FEM is described. The stress analysis of a two-dimensional rectangular plate with an edge crack under a uniform tension load, as shown in Fig. 6, is considered. An example of the distribution of enrichment nodes used in the X-FEM analysis is added to Fig. 6.

This problem has a symmetry property with respect to the $x$-axis. To simplify the description, a node enriched with the asymptotic solution of displacement near a crack tip is referred to as a $C$-node, and a node enriched with the Heaviside function is referred to as a $J$-node. The $C$-node has totally 10 degrees of freedom, which include two degrees of freedom for displacement in the $x$ and $y$ directions and four degrees of freedom for the asymptotic basis functions in each of the $x$ and $y$ directions. On the other hand, the $J$-node has totally 4 degrees of freedom, which include two degrees of freedoms for displacement in $\mathrm{x}$ and $\mathrm{y}$ directions and one degree of freedom for the Heaviside function in each of the $x$ and $y$ directions.

To consider the symmetric conditions, the analysis model, which models only the region above the $x$-axis, can be used. The symmetric model does not require the enrichment nodes on the crack with the Heaviside function. The displacement in the $y$-direction on the ligament along the symmetric axis is constrained as in the conventional FEM. On the other hand, consideration of the constraint conditions with respect to the $C$-node on the symmetric axis is required.

In order to consider the constraint conditions for $C$-nodes on the symmetric axis, the total number of degrees of freedom with respect to the basis for the $C$-node in the entire model is evaluated. As shown in Fig. 6, the element (3-4-6-5), which is located above the symmetric axis and includes just two $C$-nodes on the symmetric axis, is assumed. The displacement field $(u, v)$ at any point $(x, y)$ inside the element can be expressed as follows:

$$
\begin{aligned}
& u(x, y)=N_{3} u_{3}+N_{4} u_{4}+N_{5} u_{5}+N_{6} u_{6}+N_{3} f(r, \theta) c_{3}+N_{4} f(r, \theta) c_{4} \\
& v(x, y)=N_{3} v_{3}+N_{4} v_{4}+N_{5} v_{5}+N_{6} v_{6}+N_{3} f(r, \theta) d_{3}+N_{4} f(r, \theta) d_{4}
\end{aligned}
$$

where $u_{\mathrm{I}}, v_{\mathrm{I}}, c_{\mathrm{I}}$, and $d_{\mathrm{I}}$ are nodal freedoms, and the interpolation function $N_{\mathrm{I}}$ is described in global coordinates rather than natural coordinates. However, the enrichment function $\gamma_{\mathrm{k}}$ defined by Eq. (3) is represented by $f(r, \theta)$.

Similarly, in the entire model, the element (1-2-4-3), which is located in the symmetric position of the element (3-4-5-6) with respect to the $x$-axis, is considered. The displacement field at position $(x,-y)$, which is the symmetric position of arbitrary point $(x, y)$ in the element (3-4-6-5) can be expressed as follows:

$$
\begin{aligned}
& u(x,-y)=N_{1} u_{1}+N_{2} u_{2}+N_{3} u_{3}+N_{4} u_{4}+N_{3} f(r,-\theta) c_{3}+N_{4} f(r,-\theta) c_{4} \\
& v(x,-y)=N_{1} v_{1}+N_{2} v_{2}+N_{3} v_{3}+N_{4} v_{4}+N_{3} f(r,-\theta) d_{3}+N_{4} f(r,-\theta) d_{4}
\end{aligned}
$$

Since the displacements $u$ and $v$ should have symmetric and anti-symmetric properties with respect to $x$-axis, respectively, the following equations should be satisfied:

$$
\begin{aligned}
& u(x, y)=u(x,-y) \\
& v(x, y)=-v(x,-y)
\end{aligned}
$$

By substituting Eqs. (10a) and (11a) into Eq. (12a) and considering the symmetric properties $N_{1}=N_{5}, N_{2}=N_{6}, u_{1}=u_{5}$, and $u_{2}=u_{6}$ and by substituting Eqs. (10b) and (11b) into Eq. (12b) and considering the symmetric properties $v_{1}=-v_{5}$ and $v_{2}=-v_{6}$, Eqs. (12a) and (12b) can be written as follows:

$$
\begin{aligned}
& N_{3}\{f(r, \theta)-f(r,-\theta)\} c_{3}+N_{4}\{f(r, \theta)-f(r,-\theta)\} c_{4}=0 \\
& N_{3}\{f(r, \theta)+f(r,-\theta)\} d_{3}+N_{4}\{f(r, \theta)+f(r,-\theta)\} d_{4}=0
\end{aligned}
$$

If $f(r, \theta)$ is an even function with respect to $\theta$ or $y$, Eq. (13a) is always satisfied. On the other hand, if $f(r, \theta)$ is an odd function with respect to $\theta$ or $y, c_{3}$ and $c_{4}$ should take 0 to 
satisfy Eq. (13a) independently of $\theta$ or $y$. Similarly, if $f(r, \theta)$ is an odd function with respect to $\theta$ or $y$, Eq. (13b) is always satisfied, and $d_{3}$ and $d_{4}$ should take 0 to satisfy Eq. (13b) independently of $\theta$ or $y$. Based on the above consideration, the symmetric displacement components at the $C$-node on the symmetric axis in the symmetric model, which are the displacements $u$ in the $x$-direction, the degrees of freedom with respect to $\gamma_{2}$ and $\gamma_{4}$ should vanish. Similarly, the anti-symmetric displacement components at the $C$-node on the symmetric axis in the symmetric model, which are the displacements $v$ in the $y$-direction, the degrees of freedom with respect to $\gamma_{1}$ and $\gamma_{3}$ should vanish. Therefore, the degrees of freedom at the $C$-node on the symmetric axis should be constrained according to the aforementioned procedure.
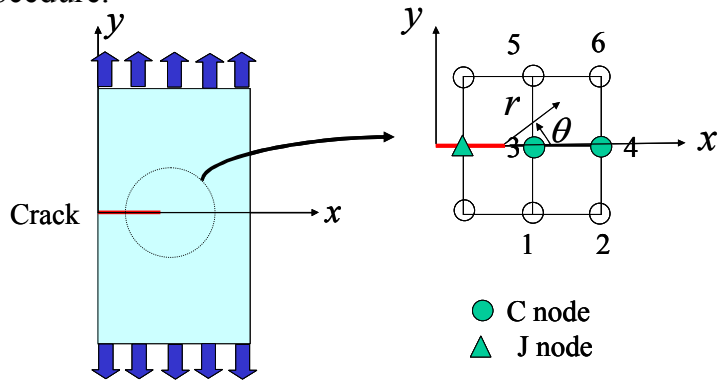

Fig.6 Plate with a edge crack under tension load

\subsection{Symmetric conditions in the three-dimensional model}

The proposed method can also be applied to a three-dimensional model. Here, a planar crack located on the plane expressed by the equation $z=0$ is assumed, and so the $x-y, \mathrm{y}-z$, and $z-x$ planes are symmetric planes. Based on Eq. (1a), the method used to enforce the constraint condition for the $C$-node on the three symmetric planes is described below.

In case the $x-y$ plane is the symmetric plane, since the displacement components $u$ and $v$ for the $C$-node on the plane are symmetric with respect to the $x-y$ plane, the degrees of freedom for the odd function, $\gamma_{2}$ and $\gamma_{4}$, with respect to $\theta$ should be constrained. Similarly, for the displacement components $w$, which are anti-symmetric with respect to the $x-y$ plane, the degrees of freedom of the odd function, $\gamma_{1}$ and $\gamma_{3}$, with respect to $\theta$ should be constrained.

In case the $y-z$ plane is the symmetric plane, since the displacement components $u$ for the $C$-node on the plane, which is perpendicular to the $y-z$ plane, should vanish for arbitrary $r$ and $\theta$, all of the degrees of freedom for the four enrichment functions $\gamma_{1}, \gamma_{2}, \gamma_{3}$, and $\gamma_{4}$ are constrained. Similarly, in case the $z-x$ plane is the symmetric plane, since the displacement components $v$ for the $C$-node on the plane, which is perpendicular to the $z-x$ plane, should vanish for arbitrary $r$ and $\theta$, all of the degrees of freedom for the four enrichment functions $\gamma_{1}, \gamma_{2}, \gamma_{3}$, and $\gamma_{4}$ are constrained These results are summarized in Table 1. In the table, the constraint condition should be given for the specified degree of freedom indicating " 0 ".

Table 1 Summary of constraint conditions for C-node on symmetric plane

\begin{tabular}{|c|c|c|c|c|}
\hline $\begin{array}{l}\text { Component of } \\
\text { displacement } \\
\text { vector }\end{array}$ & $\begin{array}{l}\text { Discription in } \\
\text { Eq.(1.1) }\end{array}$ & $\begin{array}{c}\mathrm{x}-\mathrm{y} \\
\text { plane }\end{array}$ & $\begin{array}{c}\mathrm{y}-\mathrm{z} \\
\text { plane }\end{array}$ & $\begin{array}{c}\mathrm{z}-\mathrm{x} \\
\text { plane }\end{array}$ \\
\hline $\mathrm{u}$ & $\mathrm{u}_{1}$ & --- & 0 & --- \\
\hline $\mathrm{v}$ & $\mathrm{u}_{2}$ & --- & --- & 0 \\
\hline $\mathrm{w}$ & $\mathrm{u}_{3}$ & 0 & --- & --- \\
\hline $\mathrm{u}-\gamma_{1}$ & $\mathrm{a}_{1}{ }_{1}$ & --- & 0 & --- \\
\hline $\mathrm{u}-\gamma_{2}$ & $\mathrm{a}_{2}{ }_{2}$ & 0 & 0 & --- \\
\hline $\mathrm{u}-\gamma_{3}$ & $\mathrm{a}^{1}{ }_{3}$ & --- & 0 & --- \\
\hline $\mathrm{u}-\gamma_{4}$ & $\mathrm{a}_{4}{ }_{4}$ & 0 & 0 & --- \\
\hline $\mathrm{v}-\gamma_{1}$ & $\mathrm{a}_{1}{ }_{1}$ & -- & -- & 0 \\
\hline $\mathrm{v}-\gamma_{2}$ & $\mathrm{a}_{2}{ }_{2}$ & 0 & --- & 0 \\
\hline $\mathrm{v}-\gamma_{3}$ & $\mathrm{a}_{3}{ }_{3}$ & --- & --- & 0 \\
\hline $\mathrm{v}-\gamma_{4}$ & $\mathrm{a}^{2}{ }_{4}$ & 0 & --- & 0 \\
\hline $\mathrm{w}-\gamma_{1}$ & $\mathrm{a}_{1}{ }_{1}$ & 0 & --- & --- \\
\hline $\mathrm{w}-\gamma_{2}$ & $\mathrm{a}^{3}{ }_{2}$ & --- & --- & --- \\
\hline $\mathrm{w}-\gamma_{3}$ & $\mathrm{a}_{3}{ }_{3}$ & 0 & --- & --- \\
\hline $\mathrm{w}-\gamma_{4}$ & $\mathrm{a}_{4}{ }_{4}$ & --- & --- & --- \\
\hline
\end{tabular}




\subsection{Calculation of energy release rate considering symmetric conditions}

In case the energy release rate is evaluated by the domain integral method for the symmetric model, the evaluation points may be located outside of the analyzed domain. However, the contribution of these points should be considered in order to evaluate the value properly. If an evaluation point is located outside of the domain, the contribution of the point should be considered based on the stress, strain, and displacement components at the mirror point with respect to the symmetric plane. In such a case, the signature of the components should be reversed if necessary. Note that the expression for the energy release rate by the domain integral method as shown in Eq. (5a) includes the gradient of the displacement field in addition to the stress and strain components. As shown in Fig. 7, in the half model with the $x-y$ symmetric plane, if an integration point is located below the $x-y$ plane, the stress, strain, and displacement at the point cannot be calculated directly. Therefore, the values at the mirror point with respect to the symmetric plane are used. Namely, since the normal stress component $\sigma_{x}$ and the displacement gradient $\partial u_{x} / \partial x$ are symmetric with respect to the $x-y$ plane, the following relations can be obtained:

$$
\begin{aligned}
& \sigma_{x}(x, y,-z)=\sigma_{x}(x, y, z) \\
& \partial u_{x}(x, y,-z) / \partial x=\partial u_{x}(x, y, z) / \partial x
\end{aligned}
$$

As for the normal stress components $\sigma_{y}, \sigma_{z}$ and $\tau_{x y}$ and the displacement gradient $\partial u_{y} / \partial x, \partial u_{x} / \partial y$, and $\partial u_{y} / \partial y$, similar relations can be obtained.

On the other hand, for the shear stress component $\tau_{y z}$ and $\tau_{x z}$ and the displacement gradients $\partial u_{z} / \partial x$ and $\partial u_{z} / \partial y$, because of the anti-symmetricity, the following relations can be obtained:

$$
\begin{aligned}
& \tau_{y z}(x, y,-z)=-\tau_{y z}(x, y, z) \\
& \tau_{x z}(x, y,-z)=-\tau_{x z}(x, y, z) \\
& \partial u_{z}(x, y,-z) / \partial x=-\partial u_{z}(x, y, z) / \partial x \\
& \partial u_{z}(x, y,-z) / \partial y=-\partial u_{z}(x, y, z) / \partial y
\end{aligned}
$$

The similar relations can be obtained when $y-z$ plane or $z$-x plane is symmetric plane.

Evaluating the stress and the displacement at the mirror point using Eqs. (14) and (15), the domain integral can be performed considering the symmetric conditions.

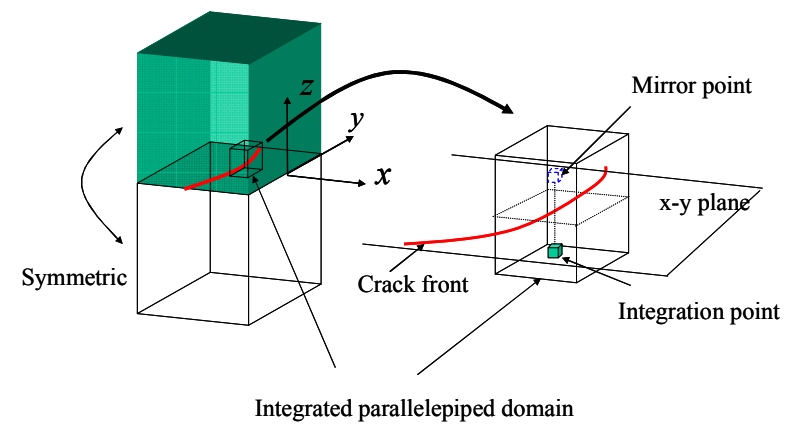

Fig.7 Domain integral for x-y symmetric half model

\section{Numerical examples}

In Section 4, elastostatic analyses including three-dimensional cracks were performed as numerical examples to verify the proposed method to introduce the symmetric conditions in X-FEM analysis. As verification problems, both a semi-elliptical surface crack and an internal circular crack were modeled. In all of the analyses, Young's modulus and the Poisson ratio for the material were set to $205.8 \mathrm{GPa}$ and 0.3 , respectively, and the analyzed domain was divided into structured eight-node hexahedral linear finite elements. Finer elements were utilized near the crack front if required. The sixth-order Gauss integration 
was adopted for the evaluation of the local stiffness for enrichment elements that contained enriched nodes, and the second-order Gauss integration was adopted for normal elements. In the process of solving the simultaneous linear equations, the conjugate gradient method with diagonal scaling was utilized, and iteration was continued until the ratio of the squared norm with respect to the residual vector to that with respect to the right-hand side vector becomes smaller than $1.0 \times 10^{-9}$. The size of the parallelepiped domain used to perform the domain integral method was properly determined depending on the problem, and the number of evaluation points was set to $50 \times 50 \times 50$ in the parallelepiped domain. Since the analyses of the present paper provide only the mode-I deformation, the obtained energy release rate can be transformed into the stress intensity factor $K_{\mathrm{I}}$ using the following equation:

$$
K_{I}=\sqrt{\frac{G E}{1-v^{2}}}
$$

where $E$ and $v$ are the Young's modulus and Poisson's ratio, respectively.

\subsection{Planar semi-elliptic surface crack}

The elastostatic analysis of the thick plate with a semi-elliptical surface crack under a uniform tension load of $\bar{p}=9.8 \mathrm{MPa}$, as shown in Fig. 8, was performed. The structured $200 \times 10 \times 40$ finite element mesh with local refinement near a crack tip was adopted. The crack modeling utilized in the X-FEM analysis is shown in Fig. 9. As shown in the figure, in order to define the curved crack front for the structured mesh division, the $C$-node is indispensable. The elastostatic analysis was performed by the analysis model, which discretizes the entire domain, and the distribution of the crack opening displacement and the stress intensity factor $K_{\mathrm{I}}$ at the crack front were evaluated. The integral region is set to the rectangular parallelepiped with the size of $40 \mathrm{~mm}$ x $20 \mathrm{~mm}$ x $40 \mathrm{~mm}$. Moreover, the analyses using the half and the quarter models were performed. An outline of the analyses including the number of nodes and elements, etc., is presented in Table 2. The distribution of the crack opening displacement at the crack end and the stress intensity factor at the crack front are shown in Fig. 10 and Fig. 11, respectively. The full, half, and quarter models were confirmed to yield the same results. Moreover, it was shown that these three analyses yielded the same results to a precision of six digits. The reference solution given by Raju et al. ${ }^{(19)}$ was added to Fig. 11. It was shown that the result obtained by X-FEM agrees well with the reference solution. Moreover, the quarter model can shorten the computation time remarkably, as shown in Table 2.
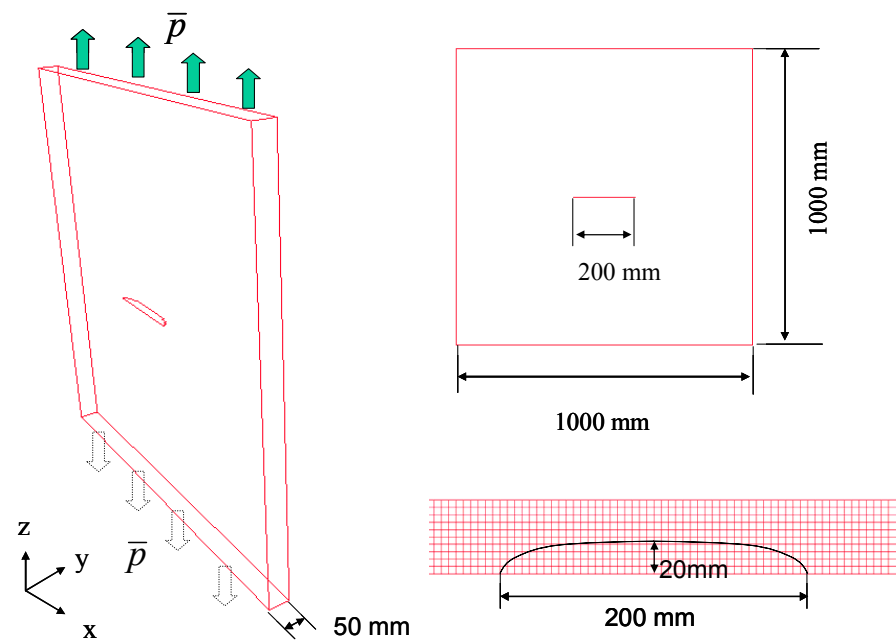

Fig.8 Planar semi-elliptical crack in a finite domain 


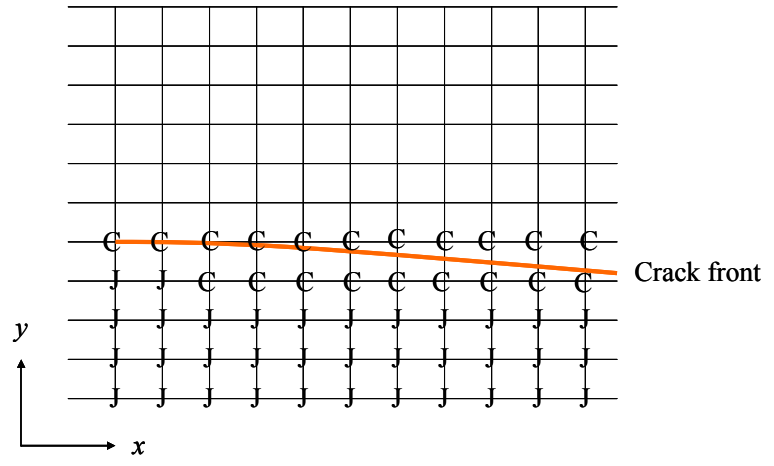

Fig.9 X-FEM modeling of a semi-elliptical crack

Table 2 Summary of X-FEM analysis for a planar semi-elliptical crack problem

\begin{tabular}{|l|c|c|c|}
\hline Model & Full & Half & Quarter \\
\hline Number of nodes & 90,651 & 46,431 & 23,331 \\
\hline Number of elements & 80,000 & 40,000 & 20,000 \\
\hline Number of DOFs & 273,070 & 137,691 & 68,964 \\
\hline Number of iterations & 2,849 & 2,732 & 2,719 \\
\hline $\begin{array}{l}\text { CPU time [sec] } \\
\text { (Pentium4/Redhat8/gcc) }\end{array}$ & 330 & 173 & 82 \\
\hline
\end{tabular}

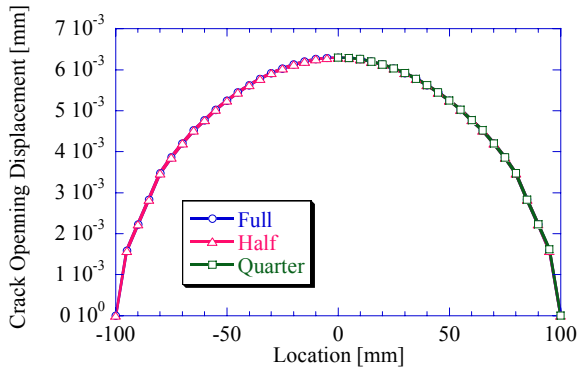

Fig.10 Distribution of crack opening displacement along $\mathrm{x}$-axis

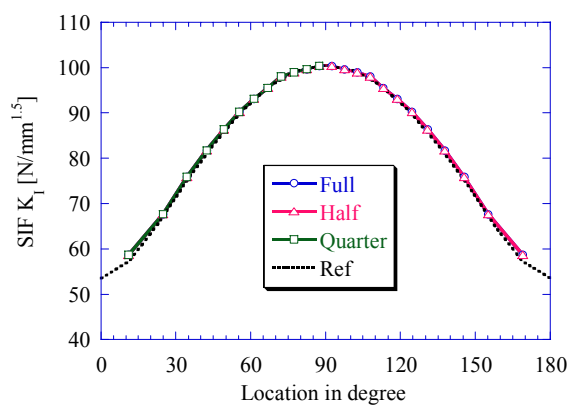

Fig.11 Distribution of stress intensity factor $K_{\mathrm{I}}$ at the front tip

\subsection{Penny-shaped crack}

The elastostatic analysis of a $2 \mathrm{~mm} \times 2 \mathrm{~mm} \times 2 \mathrm{~mm}$ finite cubed block with an internal circular crack having a radius of $0.5 \mathrm{~mm}$ under a uniform tension load of $\bar{p}=9.8 \mathrm{MPa}$, as shown in Fig. 12, was performed. A 1/8 model considering the symmetrical conditions was used. The structured finite element mesh, which has 40 divisions each in the $x, y$, and $z$ directions, was adopted. The modeling method using enrichment nodes was performed in the same manner as that for the semi-elliptical crack analysis of Fig. 9. The elastostatic analysis was performed by the analysis model to evaluate the distribution of the crack opening displacement at the center of the crack and the stress intensity factor $K_{\mathrm{I}}$ at crack front. The integral region was set as a $0.4 \mathrm{~mm} \times 0.2 \mathrm{~mm}$ x $0.4 \mathrm{~mm}$ rectangular parallelepiped. The distribution of the stress intensity factor along the circular crack front obtained by the X-FEM analysis is shown in Fig. 13, as compared with the reference 
solution by $\mathrm{Li}$ et al. ${ }^{(20)}$. The difference between the X-FEM results and the reference solution is within $1 \%$, indicating that the results obtained in the present study are appropriate.
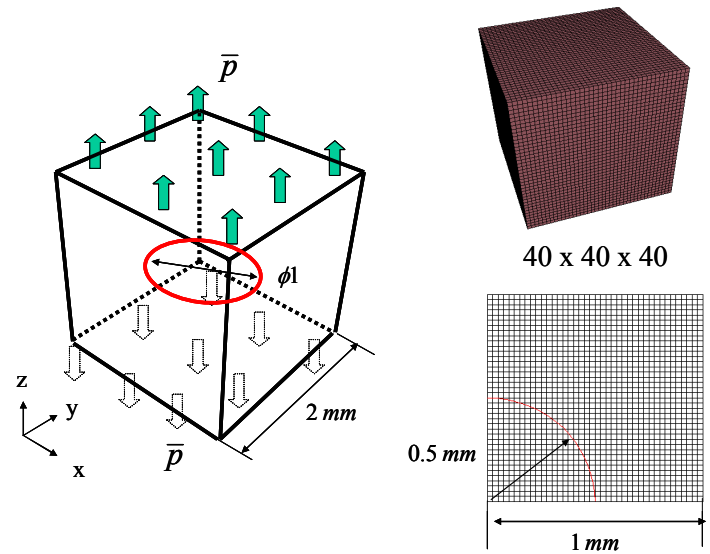

Fig.12 Penny shape crack in a finite domain

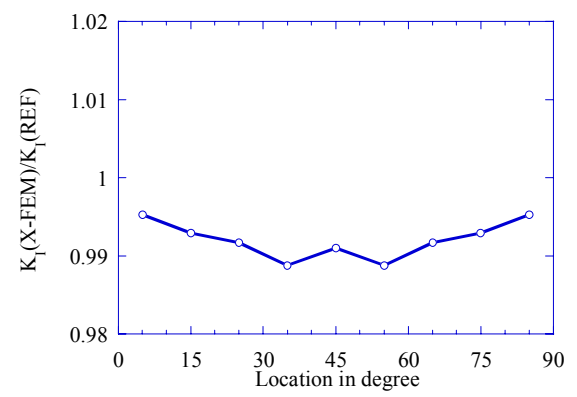

Fig.13 Distribution of stress intensity factor $K_{\mathrm{I}}$ at the front tip

\section{Conclusion}

The present paper described a method by which to perform three-dimensional X-FEM analysis using a symmetric model. Specially, the constraint conditions for the enrichment node with the asymptotic basis located on the symmetric plane were considered and the method by which to enforce the symmetric condition for three-dimensional crack problems was explicitly presented. Moreover, the evaluation method for the energy release rate by the domain integral method considering the symmetry was discussed. The proposed method was verified through stress analyses of three-dimensional planar cracks.

The proposed method by which to enforce the symmetric conditions is an indispensable procedure for the application of X-FEM to practical problems. Although the present paper describes only an elastostatic case, the proposed method can be applied to nonlinear problems because the method is only related to the essential boundary condition. In particular, as the incremental X-FEM analysis, which requires a great deal of iterative calculation, is utilized for solving nonlinear problems, the proposed method is effective for improving computational efficiency.

\section{References}

(1) Belytschko, T. and Black, T., Elastic crack growth in finite elements with minimal remeshing. Int. j. numer. methods eng., Vol. 45 (1999), pp. 602-620.

(2) Moës, N., Dolbow, J. and Belytschko, T., A finite element method for crack growth without remeshing. Int. j. numer. methods eng., Vol. 46 (1999), pp. 131-150.

(3) Sukumar, N., Moës, N., Moran, B. and Belytschko, T. , Extended finite element method for three-dimensional crack modeling. Int. j. numer. methods eng., Vol. 48 (2000), pp. 
1549-1570.

(4) Sethian, H. A., Level Set Methods and Fast Marching Methods: evolving interface in computational geometry fluid mechanics computer vision and material science. Cambridge, UK: Cambridge University Press (1999).

(5) Sukumar, N., Chopp, D. L., Moës, N. and Belytschko, T. , Modeling holes and inclusions by level sets in the extended finite element method. Comput. Methods Appl. Mech. Engrg. Vol. 190 (2001), 6183-6200.

(6) Sukumar, N., Chopp, D. L. and Moran, B., Extended finite element method and fast marching method for three-dimensional fatigue crack propagation, Engineering Fracture Mechanics, Vol. 70 (2003), pp. 29-48.

(7) Moës, N., Gravouil, A. and Belytschko, T., Non-planar 3D crack growth by the extended finite element and level sets-Part I: Mechanical model, Int. j. numer. methods eng., Vol. 53 (2002), pp. 2549-2568.

(8) Gravouil, A., Moës, N. and Belytschko, T. , Non-planar 3D crack growth by the extended finite element and level sets-Part II: Level set update. Int. j. numer. methods eng., Vol. 53 (2002), pp. 2569-2586.

(9) Nagashima, T. , Application of extended finite element method to nonlinear problems, Proceedings of WCCM VI in conjunction with APCOM'04 (2004).

(10) Legrain, G., Moës, N. and Verron E., Stress analysis around crack tips in finite strain problems using the extended finite element method, Int. j. numer. methods eng., Vol. 63(2005), pp. 290-314.

(11) Nagashima, T. and Tanaka, T., Application of the extended finite element method to elastic-plastic fracture problems, Proceedings of International Conference on Computational Methods (ICCM2004) (2004).

(12) Nagashima, T., Tanaka, T.., Application of X-FEM analysis to elastic-plastic fracture mechanics, Proceedings of the Conference on Computational Engineering and Science, Vol. 9 (2004-5), pp. 89-90.

(13) Nagashima, T., Miura, N., 3-dimensional elastic-plastic analysis by the extended finite element method, Proceedings of the Conference on Computational Engineering and Science, Vol.10 (2005-5), pp. 547-548.

(14) Rybicki, E. F. and Kanninen, M. F. , A finite element calculation of stress intensity factors by a modified crack closure integral, Engineering Fracture Mechanics, Vol. 9 (1977), pp. 931-938.

(15) Parks, D. M., A stiffness derivative finite element technique for determination of crack tip stress intensity factors, International Journal of Fracture, Vol. 10, No.4 (1974), pp. 487-502.

(16) Ikeda, T. and Sun, C. T., Stress intensity factor analysis for an interface crack between dissimilar isotropic materials under thermal stress, International Journal of Fracture, Vol. 111,(2001), pp. 229-249.

(17) Moran. B. and Shih, C. F., Crack tip and associated domain integrals from momentum and energy balance, Engineering Fracture Mechanics, Vol. 27(1987). pp. 615-642.

(18) Takahashi, Y., Nakayama, Y., Development of detailed analysis program for high-temperature crack growth evaluation, Research Report of Komae Research Laboratory, Central Research Institute of Electric Power Industry, T00044 (2001).

(19) Raju, I. S. and Newman, J. C., Jr., Eng. Fract. Mech., Vol. 11 (1979), pp. 817- 829.

(20) Li., S., Mear M.E. and Xiao, L., Symmetric weak-form integral equation method for three-dimensional fracture analysis, Compute. Methods Appli. Mech Engrg. Vol. 151(1998), pp. 435-459. 